\title{
PI3K/Akt signaling is involved in the pathogenesis of bleomycin-induced pulmonary fibrosis via regulation of epithelial-mesenchymal transition
}

\author{
XIAO-LING ZHANG ${ }^{1}$, RONG-GE XING ${ }^{1}$, LIANG CHEN $^{2}$, CHUN-RONG LIU $^{1}$ and ZHI-GANG MIAO ${ }^{1}$ \\ Departments of ${ }^{1}$ Pathology and ${ }^{2}$ Oncology, Cangzhou Central Hospital, Cangzhou, Hebei 061001, P.R. China
}

Received June 24, 2015; Accepted September 19, 2016

DOI: $10.3892 / \mathrm{mmr} .2016 .5960$

\begin{abstract}
Idiopathic pulmonary fibrosis (IPF) is a progressive lung disease characterized by chronic inflammation, fibroblast proliferation and extracellular matrix deposition. However, the molecular and cellular mechanisms underlying the pathogenesis of pulmonary fibrosis remain to be fully elucidated. The contribution of the phosphoinositide 3-kinase (PI3K)/protein kinase $\mathrm{B}(\mathrm{Akt})$ pathway in fibrotic processes remains to be investigated. The aim of the present study was to investigate the role of the PI3K/Akt pathway in pulmonary fibrosis. A rat model of pulmonary fibrosis was induced by intratracheal administration of bleomycin (BLM), and a specific PI3K/Akt inhibitor, LY294002, was used to assess the role of the PI3K/Akt pathway in fibrogenesis. The inflammatory and fibrotic alterations in the lung tissues were evaluated using histological staining and the hydroxyproline assay. In addition, the concentration of cytokines in bronchoalveolar lavage fluid and the expression of Akt, phosphorylated (p-)Akt, epithelial cadherin, $\alpha$ smooth muscle actin and vimentin in lung tissues. The data demonstrated that an increase in the expression levels of p-Akt was involved in the progression of pulmonary fibrosis and contributed to fibrogenesis. Administration of the Akt inhibitor significantly attenuated inflammation and fibrosis, which was accompanied by a reversal of lung fibrosis-associated epithelial-mesenchymal transition. Taken together, these observations suggest that the PI3K/Akt pathway serves a central role in the pathophysiology of lung fibrosis, and is a promising therapeutic target.
\end{abstract}

Correspondence to: Dr Xiao-Ling Zhang, Department of Pathology, Cangzhou Central Hospital, 16 Xinhua West Road, Cangzhou, Hebei 061001, P.R. China

E-mail: zhangxiaoling1970@yeah.net

Abbreviations: IPF, idiopathic pulmonary fibrosis; PI3K, phosphatidylinositol 3-kinase; EMT, epithelial-mesenchymal transition; BLM, bleomycin; BALF, bronchoalveolar lavage fluid; E-cad, epithelial cadherin; $\alpha$-SMA, $\alpha$ smooth muscle actin; vim, vimentin

Key words: pulmonary fibrosis, epithelial-mesenchymal transition, PI3K/Akt pathway, bleomycin, LY294002

\section{Introduction}

Idiopathic pulmonary fibrosis (IPF) is the most common type of interstitial lung disease characterized by a chronic, progressive and irreversible course with median survival of 3-5 years $(1,2)$. At present there is no efficacious treatment for lung fibrosis other than lung transplantation. Its primary pathological characteristics include persistent inflammatory cell infiltration, diffuse fibrosing alveolitis and alveolar epithelial cell injury. Fibroblasts become activated, then transition to the myofibroblastic phenotype. These cells may contribute to the extracellular matrix and collagen accumulation in the lungs, gradually replacing normal lung tissue by fibrotic scarring and honeycombing (3-5). It has been reported that lung inflammation initiates lung fibrosis, however, failure to resolve epithelial cell injury is critical to the pathogenesis of fibrosis. The molecular and cellular mechanisms underlying the pathogenesis of lung fibrosis remain to be fully understood. However, evidence in support of a role for epithelial-mesenchymal transition (EMT) in pulmonary fibrosis has been presented (6). Enhanced EMT may lead to excessive production, deposition and contraction of the extracellular matrix, and pathological fibrogenesis. However, the molecular mechanisms of EMT involved in the pathogenesis of lung fibrosis remain to be fully elucidated.

The etiology and pathogenesis of IPF remain unclear, however the characteristics of this disease can be mimicked using animal models of pulmonary fibrosis (7). Among these models of IPF, the bleomycin (BLM)-induced pulmonary fibrosis model is the most commonly used for studying the disease pathogenesis and pharmacotherapeutics (8). BLM, a widely used antineoplastic drug, causes interstitial pulmonary fibrosis in a dose-dependent manner (9). A single intratracheal administration of BLM into the lung of an animal has been reported to induce an inflammatory response, alveolar cell damage, EMT, fibroblast and myofibroblast dysplasia, in addition to extracellular matrix remodeling (10). Animal models of BLM-induced pulmonary fibrosis display similar phenotypes to those observed in patients with fibrotic lungs. Previous studies have demonstrated that BLM-induced pulmonary fibrosis is involved in the secretion of a variety of chemokines, epithelial cell apoptosis, the transforming growth factor $\beta 1$ (TGF- $\beta 1$ ) pathway and EMT (11-13).

The phosphoinositide 3-kinase (PI3K)/protein kinase B (Akt) pathway in cells, a key survival signaling pathway, regulates 
numerous biological processes through phosphoryl transfer (14). This pathway controls numerous cellular processes including protein synthesis, glucose metabolism, proliferation and differentiation (15). Its basal activity ensures cell survival and regulates the cell cycle, whereas inactivation of PI3K/Akt signaling results in cellular apoptosis (16). Additionally, the PI3K/Akt pathway, as a form of 'adaptive strategy', is involved in the immune response process of the host cell to counteract viral invasion (15). A previous study demonstrated that the $\mathrm{PI} 3 \mathrm{~K} / \mathrm{Akt}$ signaling pathway is upregulated in human diabetic nephropathy, and serves a crucial pathogenetic role in glycogen accumulation and tumor development (17). Akt is an effector kinase downstream from PI3K, which forms focal points for the development of novel therapeutics. LY294002, as a specific PI3K/Akt inhibitor, has been reported to significantly ameliorate the PI3K/Akt-mediated cellular processes by suppressing phosphorylation of Akt (18).

In the present study, the possible involvement of the PI3K/Akt pathway during BLM-induced lung fibrosis was investigated in rats. Furthermore, to identify the role of the PI3K/Akt pathway, PI3K/Akt inhibitor was used to explore the major role of the PI3K/Akt pathway in BLM-induced pulmonary fibrosis. The current study predominantly focused on investigating the molecular mechanisms underlying the pathogenesis of IPF, which will provide a potentially novel pharmacological target for the treatment of pulmonary fibrosis.

\section{Materials and methods}

Ethics statement. The present study was reviewed and approved by the Ethics Committee of Animal Care and Experimentation, Hebei Medical University (Shijiazhuang, China). The protocols were performed in accordance with the guidelines of National Institutes of Health (19). Prior to surgery and treatment, rats were anesthetized with chloral hydrate $(300 \mathrm{mg} / \mathrm{kg}$; intraperitoneal), and necessary efforts were taken to minimize suffering.

Animals and experimental design. A total of 72 adult male Sprague-Dawley rats, aged 6-8 weeks and weighing 200-220 g, were purchased from Beijing Vital River Laboratory Animal Technology Co., Ltd. (Beijing, China). The rats used for experiments were allowed free access to food and water and housed in a specific pathogen-free room. The animals were maintained on a $12 \mathrm{~h}$ light/dark cycle in a controlled temperature $\left(20-25^{\circ} \mathrm{C}\right)$ and humidity $(50 \pm 5 \%)$ environment. All rats were randomly divided into three groups: i) Intratracheal saline (control group); ii) intratracheal BLM (BLM group); iii) intratracheal BLM plus LY294002 (BLM + LY294002 group). Over the course of the experiments, the rats were closely monitored for overall health and activity.

BLM-induced pulmonary fibrosis model. For the induction of the pulmonary fibrosis model, BLM (Sanofi-Aventis, Diegem, Belgium) was dissolved in sterile phosphate-buffered saline (PBS). Subsequent to anesthesia with intraperitoneal ketamine (80 mg/kg; Zhongshan Biology \& Technology, Beijing, China) and xylazine (15 mg/kg; Zhongshan Biology \& Technology), rats were intratracheally injected with a single dose of BLM $(5.0 \mathrm{mg} / \mathrm{kg}$ body weight in $0.2 \mathrm{ml} \mathrm{PBS})$. Control animals were given a single intratracheal dose of $0.2 \mathrm{ml}$ of PBS solution only.
The rats in the LY294002 group were first treated with BLM according to the protocol described above. On the third hour following exposure to BLM, rats were treated with intratracheal administration of LY294002 (0.3 mg/kg; Cell Signaling Technology, Inc., Danvers, MA, USA). All rats were subsequently sacrificed using chloral hydrate $(300 \mathrm{mg} / \mathrm{kg}$; intraperitoneal: Baxtor, Deerfield, IL, USA) at 3, 7, 14 and 28 days subsequent to BLM treatment, and lung tissues and bronchoalveolar lavage (BAL) fluid (BALF) were collected for histopathology, collagen quantification, reverse transcription-quantitative polymerase chain reaction (RT-qPCR) and western blot analysis.

Tissue preparation and histological analysis. The lungs from the rats were dissected and fixed for $48 \mathrm{~h}$ in $4 \%$ paraformaldehyde. The fixed lungs were sectioned, embedded in paraffin, cut into $5 \mu \mathrm{m}$ sections, and stained with hematoxylin and eosin (H\&E) for analysis of lung injury, and with Elastica-Masson trichrome stain for the assessment of the collagen deposition, an index of pulmonary fibrosis. The histological severity of the lung alveolitis and lung fibrosis was scored as previously described (20).

Hydroxyproline assay. Pulmonary collagen content was determined by the measurement of hydroxyproline content. Hydroxyproline quantification was performed as previously described (21). The right lung lobes were incised and homogenized in $5 \mathrm{ml} 0.5 \mathrm{M}$ acetic acid (Invitrogen; Thermo Fisher Scientific, Inc., Waltham, MA, USA) in PBS containing $0.6 \%$ pepsin (Invitrogen; Thermo Fisher Scientific, Inc.). The extracts were rotated at $4^{\circ} \mathrm{C}$ overnight and cleared by centrifugation at $15,000 \times \mathrm{g}$ for $10 \mathrm{~min}$ at $4^{\circ} \mathrm{C}$. The hydroxyproline content was determined according to the protocol of the Hydroxyproline Testing Kit (Jiancheng, Nanjing, China). The absorbance was measured at $550 \mathrm{~nm}$ using a microplate reader.

BAL and enzyme-linked immunosorbent assay (ELISA) analysis. BAL was performed to collect BALF for analysis of the cytokine content. The rats were anesthetized with intraperitoneal ketamine $(80 \mathrm{mg} / \mathrm{kg})$ and xylazine $(15 \mathrm{mg} / \mathrm{kg})$, the trachea was then exposed and a plastic cannula was inserted into the trachea. The lung tissues were lavaged with $5 \mathrm{ml} 0.9 \%$ saline solution. The lavage was repeated twice with saline to recover a total volume of 4-5 $\mathrm{ml}$. It was then centrifuged at $500 \mathrm{x} \mathrm{g}$ for $10 \mathrm{~min}$ at $4^{\circ} \mathrm{C}$ and the supernatant was used for cytokine measurement. The concentrations of tumor necrosis factor (TNF)- $\alpha$, interleukin (IL)-1 $\beta$, IL- 6 and IL-10 in the BALF were determined using ELISA kits (R\&D Systems, Inc., Minneapolis, MN, USA) according to the manufacturer's instructions.

RNA isolation and RT-qPCR analysis. Total RNA was isolated from rat lung tissues using TRIzol reagent (Life Technologies; Thermo Fisher Scientific, Inc.) and reverse-transcribed using the First Strand cDNA synthesis kit (Fermentas; Thermo Fisher Scientific, Inc., St. Leon-Rot, Germany) according to the manufacturer's instructions. RT-qPCR was performed on a RT-qPCR System (ABI Prism 7300; Applied Biosystems; Thermo Fisher Scientific, Inc.) instrument with SYBR Premix Ex Taq (Takara Bio., Inc., Otsu, Japan), starting with 1 ng reverse-transcribed total RNA. PCR was performed under the following conditions: $95^{\circ} \mathrm{C}$ for $10 \mathrm{~min}, 40$ cycles of $95^{\circ} \mathrm{C}$ for $5 \mathrm{sec}$ and $60^{\circ} \mathrm{C}$ for $30 \mathrm{sec}$. 

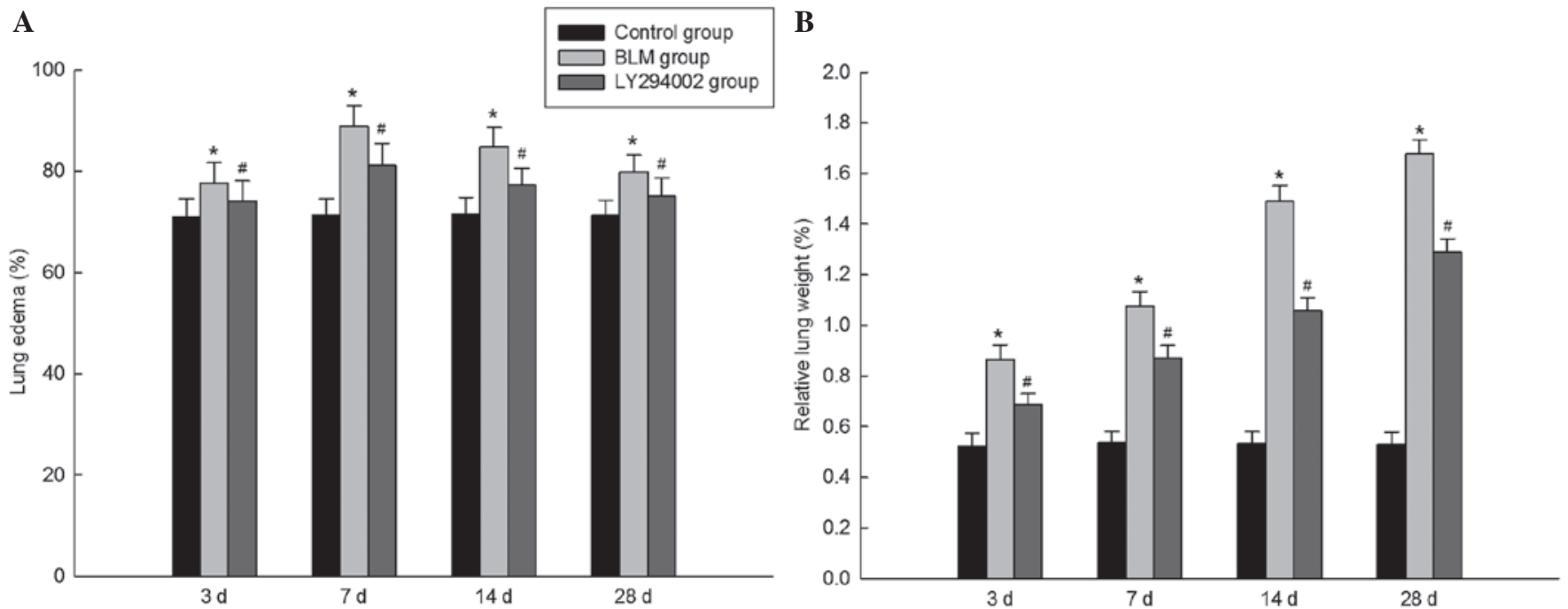

Figure 1. The effect of protein kinase B inhibitor on lung edema and weight subsequent to BLM exposure. Lungs were collected at 3,7 , 14 and 28 days subsequent to BLM and were weighed. (A) Alterations in the levels of lung edema in the different groups. (B) Alterations in relative lung weight in different groups. Bars represent the mean \pm standard error $(n=6)$. ${ }^{*} \mathrm{P}<0.05$ vs. control group and ${ }^{\#} \mathrm{P}<0.05$ vs. BLM group at the same time points. BLM, bleomycin.

The following primers were used: TNF- $\alpha$, Forward (F) 5'-TTG ACCTCAGCGCTGAGTTG-3' and reverse (R) 5'-CCTGTA GCCCACGTCGTAGC-3'; IL-1 $\beta$, F 5'-CAGGATGAGGAC ATGAGCACC-3' and R 5'-CTCTGCAGACTCAAACTC CAC-3'; IL-6, F 5'-GTACTCCAGAAGACCAGAGG-3' and R 5'-TGCTGGTGACAACCACGGCC-3'; IL-10, F 5'-CAT GGCCTTGTAGACACCTTTG-3' and R 5'-CATCGATTTCTC CCCTGTGAGA-3'. GAPDH was used as an internal control. Each experiment was performed in duplicate and repeated three times. The data were analysed using the $2^{-\Delta \Delta \mathrm{Cq}}$ method (22).

Western blot analysis. Lung tissues were lysed in Tissue Protein Lysis Solution (Life Technologies; Thermo Fisher Scientific, Inc., Gent, Belgium) supplemented with 5\% Proteinase Inhibitor Cocktail (Sigma-Aldrich; Merck Millipore, Darmstadt, Germany), incubated on ice for $30 \mathrm{~min}$, and centrifuged at $15,000 \times \mathrm{g}$ for $15 \mathrm{~min}$ at $4^{\circ} \mathrm{C}$. Protein concentrations were determined with the bicinchoninic acid protein assay reagents (Jiancheng, Nanjing, China). Proteins from each sample were separated on $10 \%$ sodium dodecyl sulfate-polyacrylimide gel electrophoresis, transferred to polyvinylidene difluoride membranes for $60 \mathrm{~min}$. Nonspecific binding sites were blocked with $5 \%$ bovine serum albumin (Sigma-Aldrich; Merck Millipore) for $1 \mathrm{~h}$, then incubated with the following rabbit anti-rat polyclonal antibodies: Akt (cat. no. sc-32245), p-Akt (cat. no. sc-20984), epithelial cadherin (E-cad; cat. no. sc-30956), $\alpha$-smooth muscle actin ( $\alpha$-SMA; cat. no. sc-28538), vimentin (Vim; cat. no. sc-31638) and $\beta$-actin (cat. no. sc-26351; all 1:1,000; Santa Cruz Biotechnology, Inc., Santa Cruz, CA, USA) overnight at $4^{\circ} \mathrm{C}$. Next day, the membranes were incubated with secondary antibodies (cat. no. IBSBIOA-003; 1:5,000; Cell Signaling Technology, Inc.) at $37^{\circ} \mathrm{C}$ for $2 \mathrm{~h}$. The immunoreactive bands were visualized with an enhanced chemiluminescent detection system (Amersham, Little Chalfont, UK). Blots were scanned by densitometry, and the integrated density of pixels was quantified using ImageQuant software, version 5.2 (GE Healthcare Bio-Sciences, Pittsburgh, PA, USA).
Statistical analysis. Data are expressed as the means \pm standard deviation. All tests were performed using SPSS software, version 17.0 (SPSS, Inc., Chicago, IL, USA). Statistical significance was determined using one-way analysis of variance and the Student-Newmann-Keuls post hoc test to determine differences among different groups. $\mathrm{P}<0.05$ was considered to indicate a statistically significant difference.

\section{Results}

Administration of Akt inhibitor alleviates BLM-induced lung edema and weight. As presented in Fig. 1, clear lung edema was observed in BLM-challenged rats, at the same time, the relative weights of the lungs were significantly increased in the BLM group rats. Notably, inhibition of p-Akt, using tracheal administration of LY294002, significantly alleviated BLM-induced lung edema and elevation of the relative lung weight.

Administration of Akt inhibitor reduces BLM-induced inflammation. To investigate the role of the PI3K/Akt pathway on BLM-induced pulmonary inflammation and fibrosis, the effects of the Akt inhibitor on the histopathological alterations observed in the lungs were observed. Fig. 2 presents the H\&E-stained lung sections. A histological evaluation indicated that there were no changes in the control rats, whereas the section from the BLM group revealed disordered lung tissue structure, thickened pulmonary interalveolar septa and infiltrated inflammatory cells. In contrast, the BLM-induced histological alterations were significantly reduced by treatment with the Akt inhibitor according to the histological observations.

Administration of the Akt inhibitor attenuates BLM-induced fibrosis. Masson's trichrome staining indicated that there were large-scale collagen accumulations in the BLM group (Fig. 3A). Excessive deposition of extracellular matrix was observed in the lung tissues of rats in the BLM group, which is in accordance with the hallmark characteristics of pulmonary fibrosis (Fig. 3A). Administration of the Akt inhibitor significantly 
A

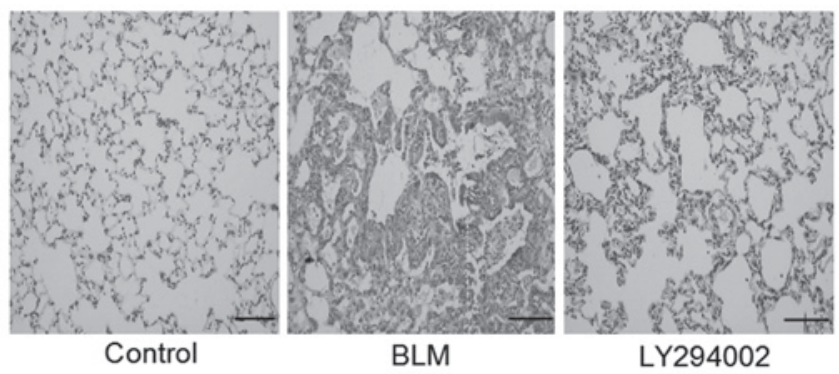

B

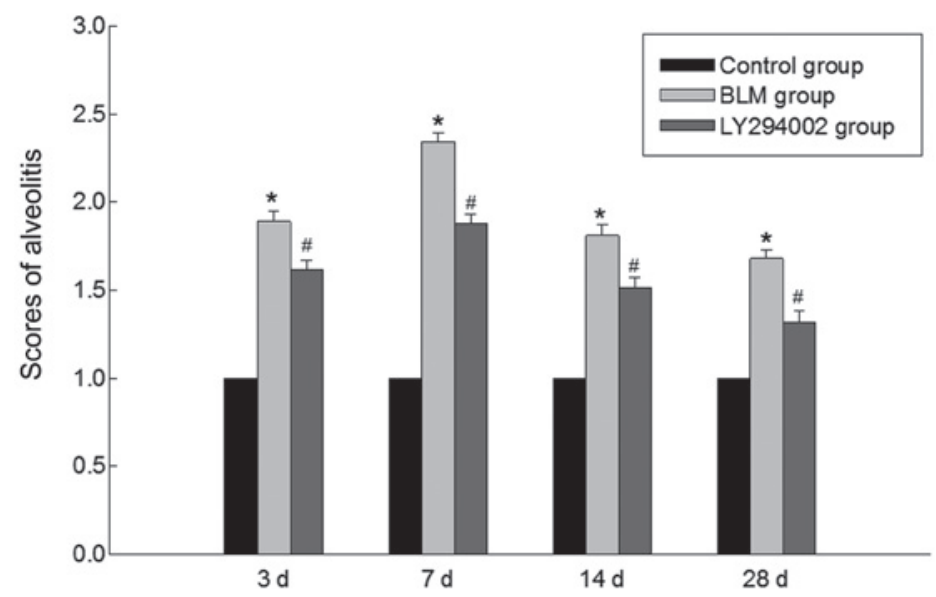

Figure 2. The effect of the protein kinase B inhibitor on alveolitis subsequent to BLM exposure. (A) Representative images of hematoxylin and eosin staining for lung tissues at 7 days subsequent to BLM treatment. Original magnification, $x 400$. (B) Pathohistological scores of lung sections were evaluated according to pulmonary inflammation. Error bars represent the mean \pm standard error $(\mathrm{n}=6)$. ${ }^{*} \mathrm{P}<0.05$ vs. control group and ${ }^{\#} \mathrm{P}<0.05$ vs. BLM group at the same time points. BLM, bleomycin.

suppressed BLM-induced matrix protein deposition in the lungs of BLM-treated rats. Similarly, a significant reduction in the fibrosis scoring of these sections was observed in the rat lungs of the BLM + LY294002 group (Fig. 3B). Furthermore, hydroxyproline is suggested to be able to act as a primary indicator of the collagen metabolism in different tissues. In order to more accurately illustrate the severity of lung fibrosis, the content of hydroxyproline was assessed to indicate collagen accumulation within the fibrotic lung tissues. As presented in Fig. 3C, the hydroxyproline content in the BLM group was significantly greater than that of the BLM + LY294002 group, indicating that the Akt inhibitor significantly reduced BLM-induced elevation of hydroxyproline content in the lung tissues. In addition, the gene expression levels of collagen I and III were measured, and the results indicated a similar trend to that of the hydroxyproline results (Fig. 3D and E).

Administration of the Akt inhibitor upregulates anti-inflammatory cytokines and downregulates pro-inflammatory cytokines. The levels of TNF- $\alpha$, IL-1 $\beta$, IL-6 or IL-10 in the BALF of the rats were detected via ELISA. As presented in Fig. 4, in the BLM group, the concentrations of TNF- $\alpha$, IL- $1 \beta$ and IL- 6 in the BALF were significantly increased, while IL-10 levels in the BALF were significantly reduced compared with the control group. However, treatment with the Akt inhibitor could reverse the increases in TNF- $\alpha$, IL-1 $\beta$ and IL-6 levels and the reductions in the IL-10 levels in the BALF. The results demonstrated that the Akt inhibitor may downregulate pro-inflammatory cytokines whereas may upregulate anti-inflammatory cytokines. Therefore, it is hypothesized that the PI3K/Akt pathway is involved in the pathogenesis of BLM-induced pulmonary fibrosis by influencing the expression of inflammatory factors.

The relative expression of proteins in the PI3K/Akt signaling pathway. The protein expression levels of Akt and p-Akt were assessed in lung tisses using western blot analysis. The molecular weight of the Akt protein is $55 \mathrm{kDa}$ and of the $\mathrm{p}$-Akt protein is $60 \mathrm{kDa}$. As presented in Fig. 5, BLM treatment markedly increased the phosphorylation level of Akt protein, while no significant alterations were observed in the levels of total Akt protein. The expression levels of p-Akt were the greatest 7 days subsequent to BLM injection. The increased phosphorylation of Akt in lung tissue following BLM challenge was significantly reduced in the BLM + LY294002 group. These results indicated that the PI3K/Akt signaling pathway was involved in the pathogenesis of BLM-induced pulmonary fibrosis.

\section{Administration of Akt inhibitor reverses BLM-induced EMT. In} order to investigate the involvement of the PI3K/Akt pathway in mediating BLM-induced EMT, the epithelial marker E-cad and the mesenchymal markers $\alpha$-SMA and Vim were analyzed by RT-qPCR and western blotting. The molecular weight of the E-cad protein is $120 \mathrm{kDa}$, for the $\alpha$-SMA protein is $42 \mathrm{kDa}$ and for Vim protein is $57 \mathrm{kDa}$. As presented in Fig. 6, E-cad expression was downregulated in the lungs following BLM stimulation, while the expression of $\alpha$-SMA and Vim were upregulated. Notably, blocking of the PI3K/Akt pathway significantly increased the expression of epithelial marker genes and proteins, whereas the expression of mesenchymal 
A

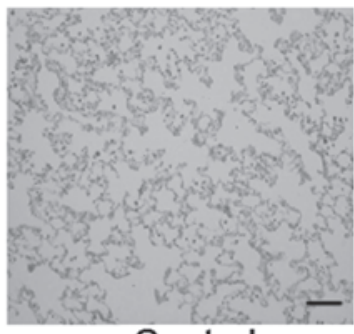

Control

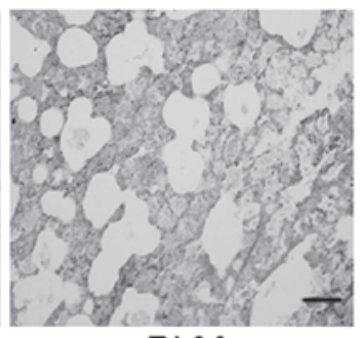

BLM

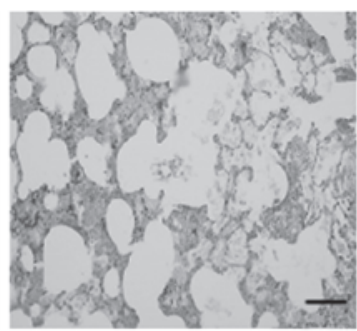

LY294002
B

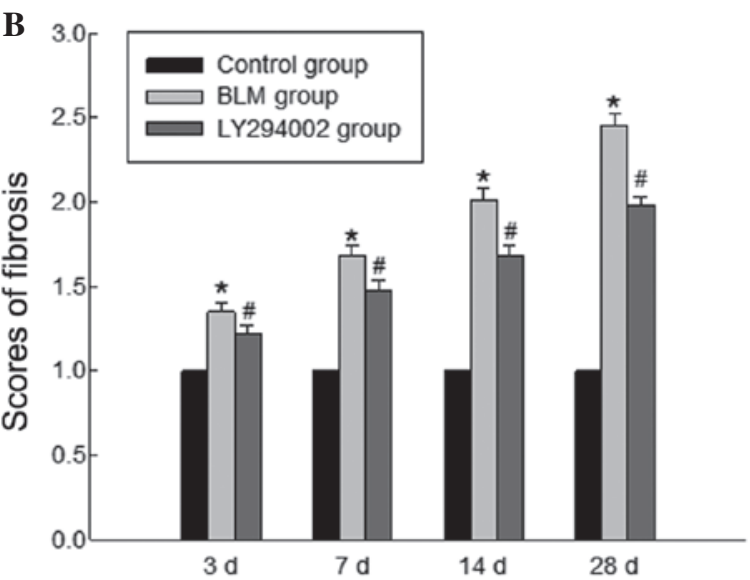

D

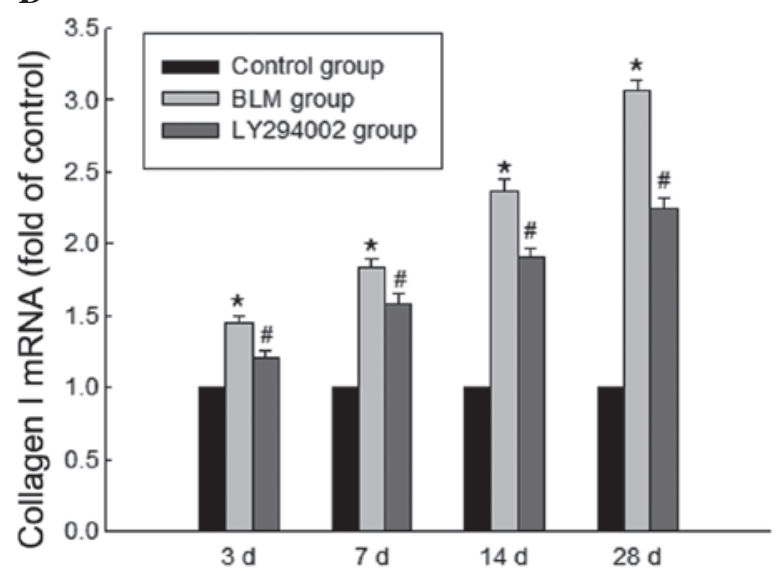

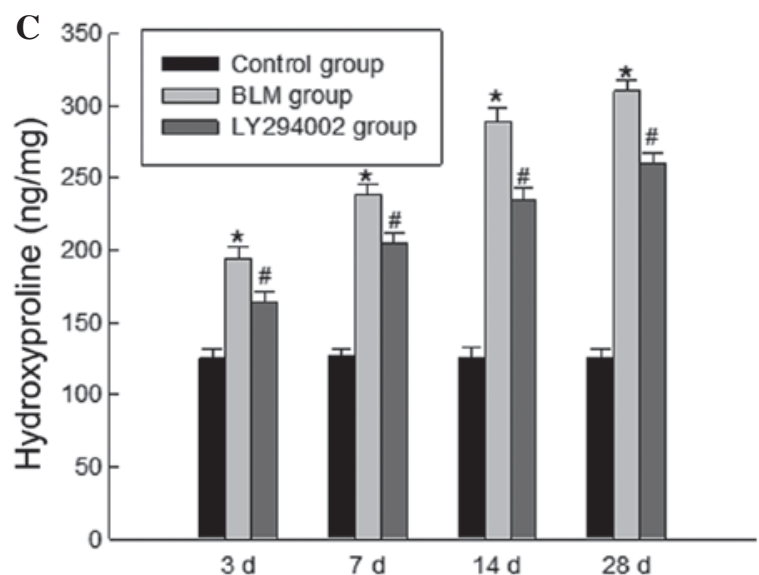

E

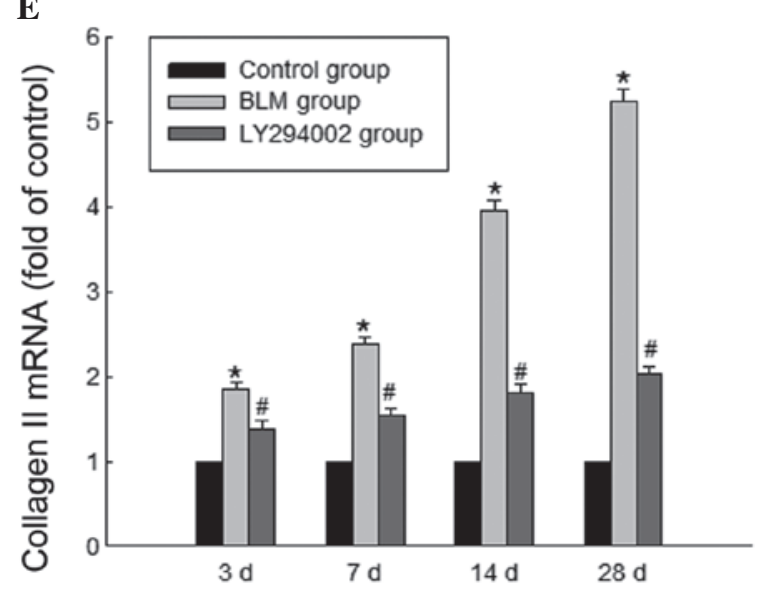

Figure 3. The effect of the protein kinase B inhibitor on pulmonary fibrosis subsequent to BLM exposure. (A) Representative images of Masson's trichrome staining of lung sections obtained from the rat lungs. Original magnification, $x 400$. (B) The fibrosis scores of lung sections were evaluated according to pulmonary fibrosis. (C) Quantitation of collagen accumulation in the lungs was analyzed using hydroxyproline assay. Reverse transcription-quantitative polymerase chain reaction analysis for (D) collagen I and (E) collagen III mRNA expression in the different groups. Error bars represent the mean \pm standard error ( $\mathrm{n}=6$ ). ${ }^{*} \mathrm{P}<0.05$ vs. control group and ${ }^{\#} \mathrm{P}<0.05$ vs. BLM group at the same time points. BLM, bleomycin.

marker genes and proteins were reduced. Taken together, these results indicate that the Akt inhibitor attenuated fibrosis subsequent to BLM treatment, potentially via inhibition of BLM-induced EMT.

\section{Discussion}

The PI3K/Akt signaling pathway in the host cell serves a critical regulatory role in numerous cellular processes including translation, metabolism, RNA processing, apoptosis and autophagy (15). A previous study demonstrated that the activation of the PI3K/Akt pathway is a crucial molecular event in a variety of diseases, including in cancer development and maintenance via the regulation of cell survival, cellular growth and cell cycle progression (23). In the present study, male Sprague-Dawley rats were subjected to intratracheal injection of BLM $(5 \mathrm{mg} / \mathrm{kg} ; 0.2 \mathrm{ml})$ to induce the pulmonary fibrosis model. The results of western blotting demonstrated that BLM administration markedly upregulated the phosphorylation level of Akt protein, while no significant alterations in the total Akt protein level were observed, indicating that the PI3K/Akt pathway is invovled in lung fibrosis. In the current study, LY294002, a specific Akt inhibitor, was used to further identify the roles of the PI3K/Akt pathway in the pathogenesis of pulmonary fibrosis. The data indicated that inhibition of the PI3K/Akt pathway may markedly block BLM-induced increases in p-Akt expression, while no effects on Akt protein levels in the lung tissues were observed. 

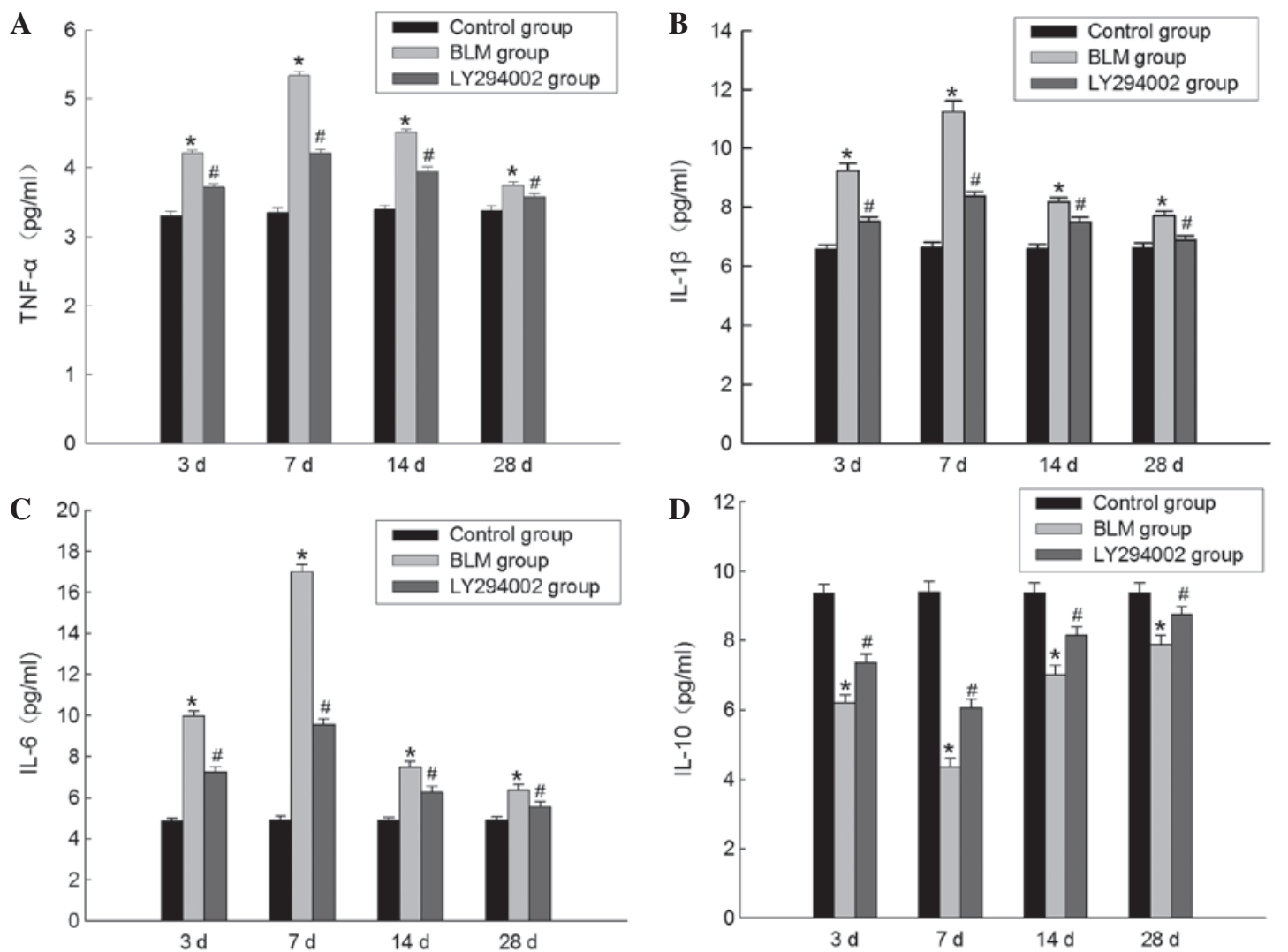

Figure 4. Effects of the protein kinase B inhibitor on inflammatory cytokines in BALF following BLM exposure. Concentrations of (A) TNF- $\alpha$, (B) IL-1 $\beta$, (C) IL-6 or (D) IL-10 in BALF of the different groups were measured by an enzyme-linked immunosorbent assay. Bars indicate mean \pm standard error ( $\mathrm{n}=6$ ). ${ }^{*} \mathrm{P}<0.05$ vs. control group and ${ }^{\#} \mathrm{P}<0.05$ vs. BLM group at the same time point. BALF, bronchoalveolar lavage fluid; BLM, bleomycin; TNF- $\alpha$, tumor necrosis factor $\alpha$; IL, interleukin.

A
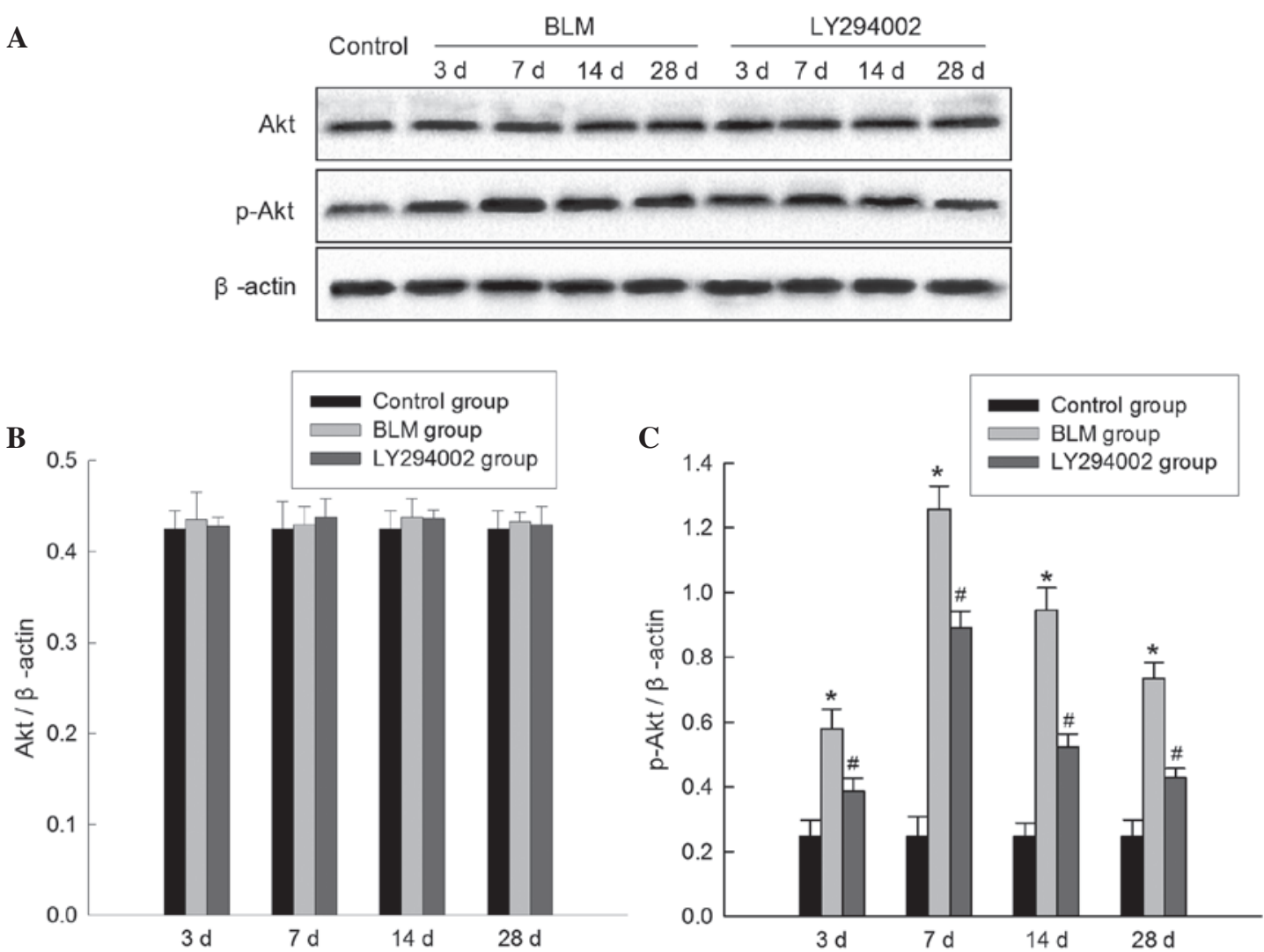

Figure 5. The expression of PI3K/Akt signaling pathway-associated proteins in the lung tissues. (A) Western blot analysis of Akt and p-Akt bands in the lungs. (B) Akt and (C) p-Akt levels were quantified by densitometry and normalized to $\beta$-actin. Bars represent the mean \pm standard error $(\mathrm{n}=6)$. ${ }^{*} \mathrm{P}<0.05 \mathrm{vs}$. control group and "P<0.05 vs. BLM group at the same time points. PI3K, phosphoinositide 3-kinase; Akt, protein kinase B; p-, phosphorylated; BLM, bleomycin. 
A
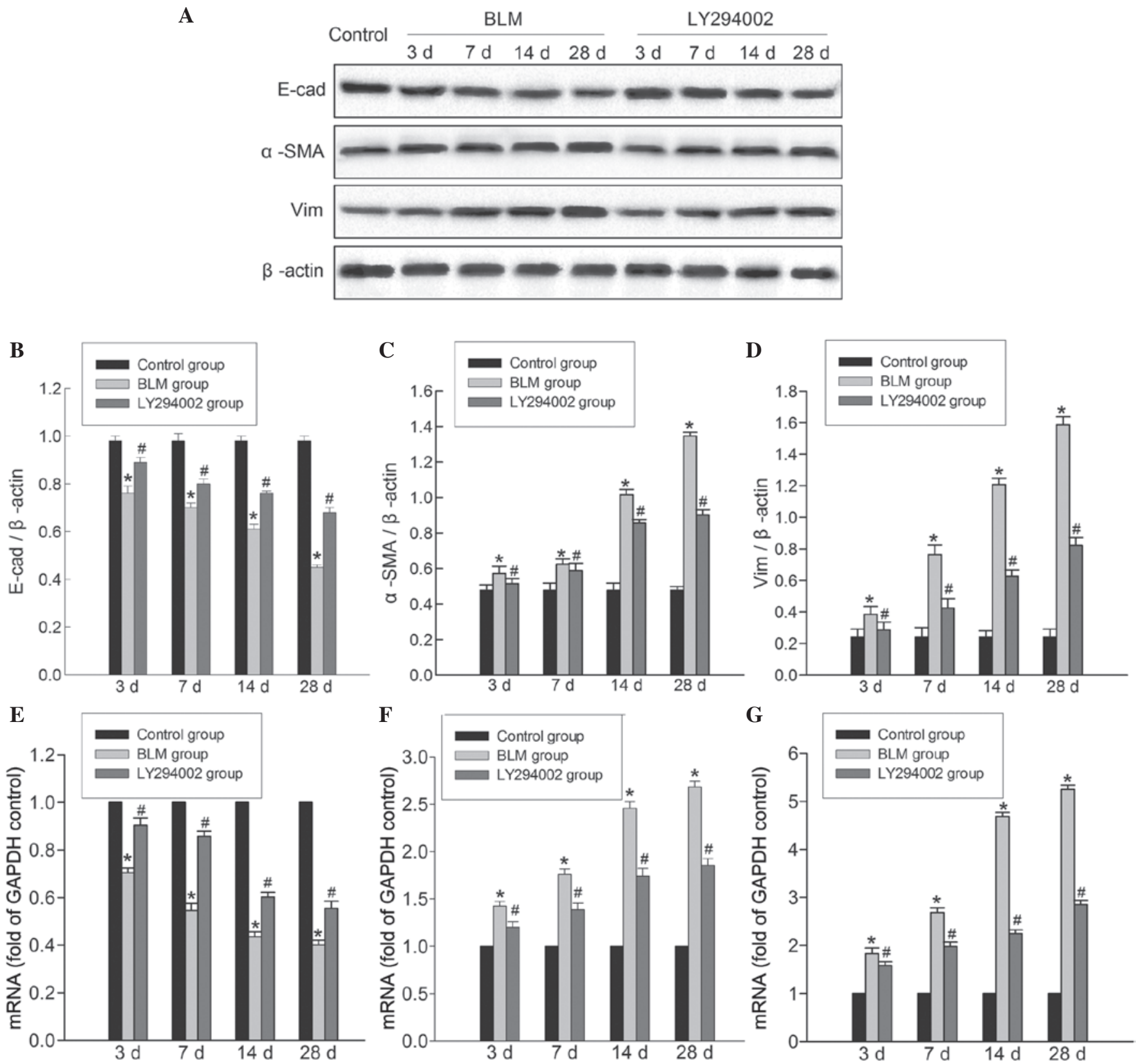

Figure 6. The effect of the protein kinase B inhibitor on epithelial-mesenchymal transition subsequent to BLM exposure. (A) Western blot analysis for E-cad, $\alpha$-SMA and Vim protein expression. (B-D) The bands were quantified by densitometry and normalized to $\beta$-actin. Reverse transcription-quantitative polymerase chain reaction analysis for (E) E-cad, (F) $\alpha$-SMA and (G) Vim mRNA expression and normalized to GAPDH mRNA expression. Bars represent the mean \pm standard error $(\mathrm{n}=6) .{ }^{*} \mathrm{P}<0.05$ vs. control group and ${ }^{\#} \mathrm{P}<0.05$ vs. BLM group at the same time points. BLM, bleomycin; E-cad, epithelial cadherin; $\alpha$-SMA, $\alpha$ smooth muscle actin; Vim, vimentin; GAPDH, glyceraldehyde 3-phosphate dehydrogenase.

In addition, the effects of the Akt inhibitor on BLM-induced inflammation and fibrosis were investigated. The inflammatory alterations were evaluated with $\mathrm{H} \& \mathrm{E}$ staining of lung tissues and via ELISA in BALF. The histopathological observations indicated that treatment with the Akt inhibitor ameliorated inflammatory cell infiltration and promoted lung injury repair. In addition, inhibition of the PI3K/Akt pathway could suppress the overproduction of proinflammatory cytokines including TNF- $\alpha$, IL-1 $\beta$ and IL- 6 in the BALF, and enhanced the release of the anti-inflammatory cytokine IL-10. These observations indicated that blocking the PI3K/Akt pathway exhibited positive anti-inflammatory activity in the development of BLM-induced pulmonary fibrosis. Additionally, the fibrotic alterations to lung tissue following LY294002 treatment together with BLM were observed. The results indicated that the Akt inhibitor suppressed myofibroblast expansion and fibronectin matrix formation in lung tissues, reduced the collagen content, reduced the increase of collagen I and collagen III levels, and preserved pulmonary compliance. The suggested that the Akt inhibitor exerts an anti-fibrotic effect in pulmonary fibrosis. Therefore, it was concluded that the PI3K/Akt pathway served a crucial role in BLM-induced pulmonary fibrosis, via the regulation of inflammation and fibrosis.

Whether the PI3K/Akt pathway influences lung fibrosis-associated EMT remains unclear and requires further investigation. A previous study demonstrated that EMT is associated with liver 
fibrosis and regeneration (24). An additional study observed that EMT, a phenotypic change in which epithelial cells acquire mesenchymal characteristics, served a specific role in fibrogenesis and in the progression of cancer by biophysical signaling mechanisms (25). It has been previously demonstrated in in vitro studies and animal models that TGF- $\beta$ leads to the induction of EMT in the genetically engineered type II alveolar epithelial cell line RLE/Abca3 and in BLM-induced pulmonary fibrosis in mice $(26,27)$. The present study was conducted to examine the possibility that the PI3K/Akt pathway modulated BLM-induced EMT. EMT is activated in lung tissues in BLM-challenged rats, characterized by the loss of epithelial characteristics (E-cad) and the acquisition of a mesenchymal phenotype ( $\alpha$-SMA and Vim). Previous studies have identified that the Akt inhibitor reversed the reductions of E-cad, and prevented the increased expression of $\alpha$-SMA and Vim that induce fibrosis-associated EMT (28-30). Therefore, it is suggested that EMT of alveolar epithelial cells occurs in the pathogenesis of lung fibrosis and is partly reversed by the Akt inhibitor, indicating that the PI3K/Akt pathway may promote EMT progress, contributing to the pathogenesis of fibrosis.

In conclusion, the results of the current study suggest an involvement of the PI3K/Akt signaling pathway in the pathogenesis of pulmonary fibrosis, contributing to fibrogenesis. Blocking the PI3K/Akt pathway could attenuate BLM-induced inflammation and fibrosis and reverse the process of lung fibrosis-associated EMT. Thus, pharmacological blockade of the PI3K/Akt pathway may be considered as a potential therapeutic strategy for the treatment of pulmonary fibrosis.

\section{References}

1. Guiot J, Corhay JL and Louis R: Idiopathic pulmonary fibrosis Rev Med Liege 69: 605-610, 2014.

2. Kim HJ, Perlman D and Tomic R: Natural history of idiopathic pulmonary fibrosis. Respir Med 109: 661-670, 2015.

3. Gharaee-Kermani M,Gyetko MR,Hu B and Phan SH: New insights into the pathogenesis and treatment of idiopathic pulmonary fibrosis: A potential role for stem cells in the lung parenchyma and implications for therapy. Pharm Res 24: 819-841, 2007.

4. Fernandez IE and Eickelberg O: New cellular and molecular mechanisms of lung injury and fibrosis in idiopathic pulmonary fibrosis. Lancet 380: 680-688, 2012.

5. King TE Jr, Pardo A and Selman M: Idiopathic pulmonary fibrosis. Lancet 378: 1949-1961, 2011.

6. Yan W, Xiaoli L, Guoliang A Zhonghui Z, Di L, Ximeng L, Piye N, Li C and Lin T: SB203580 inhibits epithelial-mesenchymal transition and pulmonary fibrosis in a rat silicosis model. Toxicol Lett 259: 28-34, 2016.

7. Harrison JH Jr and Lazo JS: High dose continuous infusion of bleomycin in mice: A new model for drug-induced pulmonary fibrosis. J Pharmacol Exp Ther 243: 1185-1194, 1987.

8. Moeller A, Ask K, Warburton D, Gauldie J and Kolb M: The bleomycin animal model: A useful tool to investigate treatment options for idiopathic pulmonary fibrosis? Int J Biochem Cell Biol 40: 362-382, 2008.

9. Adamson IY and Bowden DH: The pathogenesis of bleomycin-induced pulmonary fibrosis in mice. Am J Pathol 77: 185-197, 1974.

10. Moore BB and Hogaboam CM: Murine models of pulmonary fibrosis. Am J Physiol Lung Cell Mol Physiol 294: L152-L160, 2008.

11. Kim HS, Go H, Akira S and Chung DH: TLR2-mediated production of IL-27 and chemokines by respiratory epithelial cells promotes bleomycin-induced pulmonary fibrosis in mice. J Immunol 187: 4007-4017, 2011.
12. Degryse AL, Tanjore $\mathrm{H}, \mathrm{Xu} \mathrm{XC}$, Polosukhin VV, Jones BR, Boomershine CS, Ortiz C, Sherrill TP, McMahon FB, Gleaves LA, et al: TGF $\beta$ signaling in lung epithelium regulates bleomycin-induced alveolar injury and fibroblast recruitment. Am J Physiol Lung Cell Mol Physiol 300: L887-L897, 2011.

13. Chen YL, Zhang X, Bai J, Gai L, Ye XL, Zhang L, Xu Q, Zhang YX, Xu L, Li HP and Ding X: Sorafenib ameliorates bleomycin-induced pulmonary fibrosis: Potential roles in the inhibition of epithelial-mesenchymal transition and fibroblast activation. Cell Death Dis 4: e665, 2013.

14. Yin MM, Cui YR, Wang L, Wang JY, Gao Y and Xi JY: Progress on PI3K/Akt signaling pathway regulating self-renewal and pluripotency of embryonic stem cells. Sheng Li Xue Bao 66: 223-230, 2014 (In Chinese).

15. Diehl N and Schaal H: Make yourself at home: Viral hijacking of the PI3K/Akt signaling pathway. Viruses 5: 3192-3212, 2013.

16. Diab S, Fidanzi C, Léger DY, Ghezali L, Millot M, Martin F, Azar R, Esseily F, Saab A, Sol V, et al: Berberis libanotica extract targets $\mathrm{NF}-\kappa \mathrm{B} / \mathrm{COX}-2$, PI3K/Akt and mitochondrial/caspase signalling to induce human erythroleukemia cell apoptosis. Int J Oncol 47: 220-230, 2015.

17. Ribback S, Cigliano A, Kroeger N, Pilo MG, Terracciano L, Burchardt M, Bannasch P, Calvisi DF and Dombrowski F: $\mathrm{PI} 3 \mathrm{~K} / \mathrm{AKT} / \mathrm{mTOR}$ pathway plays a major pathogenetic role in glycogen accumulation and tumor development in renal distal tubules of rats and men. Oncotarget 30: 13036-13048, 2015.

18. Xu X, Li H, Hou X, Li D, He S, Wan C, Yin P, Liu M, Liu F and $\mathrm{Xu} \mathrm{J}$ : Punicalagin induces Nrf2/HO-1 expression via upregulation of PI3K/AKT pathway and inhibits LPS-induced oxidative stress in RAW264.7 macrophages. Mediators Inflamm 2015: 380218, 2015.

19. Chen H, Mei YQ and Solomon MA: Experiences of establishing an abdominal heart transplantation model in rats. Zhonghua Yi Xue Za Zhi 92: 1715-1718, 2012. (In Chinese)

20. Szapiel SV, Elson NA, Fulmer JD, Hunninghake GW and Crystal RG: Bleomycin-induced interstitial pulmonary disease in the nude, athymic mouse. Am Rev Respir Dis 120: 893-899, 1979.

21. Huang LS, Berdyshev E, Mathew B, Fu P, Gorshkova IA, He D, Ma W, Noth I, Ma SF, Pendyala S, et al: Targeting sphingosine kinase 1 attenuates bleomycin-induced pulmonary fibrosis. FASEB J 27: 1749-1760, 2013.

22. Livak KJ and Schmittgen TD: Analysis of relative gene expression data using real-time quantitative PCR and the 2(-Delta Delta C(T)) method. Methods 25: 402-408, 2001.

23. Mabuchi S, Kuroda H, Takahashi R and Sasano T: The $\mathrm{PI} 3 \mathrm{~K} / \mathrm{AKT} / \mathrm{mTOR}$ pathway as a therapeutic target in ovarian cancer. Gynecol Oncol 137: 173-179, 2015.

24. Lee SJ, Kim KH and Park KK: Mechanisms of fibrogenesis in liver cirrhosis: The molecular aspects of epithelial-mesenchymal transition. World J Hepatol 6: 207-216, 2014.

25. O'Connor JW and Gomez EW: Biomechanics of TGF $\beta$-induced epithelial-mesenchymal transition: Implications for fibrosis and cancer. Clin Transl Med 3: 23, 2014.

26. Takano M, Yamamoto C, Yamaguchi K, Kawami M and Yumoto R: Analysis of TGF- $\beta 1$ - and drug-induced epithelial-mesenchymal transition in cultured alveolar epithelial cell line RLE/Abca3. Drug Metab Pharmacokinet 30: 111-118, 2015.

27. PLOS ONE Staff: Correction: Melatonin inhibits endoplasmic reticulum stress and epithelial-mesenchymal transition during bleomycin-induced pulmonary fibrosis in mice. PloS One 10: e0119381, 2015.

28. Zhou N, Lu F, Liu C, Xu K, Huang J, Yu D and Bi L: IL-8 induces the epithelial-mesenchymal transition of renal cell carcinoma cells through the activation of AKT signaling. Oncol Lett 12: 1915-1920, 2016.

29. Shan B, Man H, Liu J, Wang L, Zhu T, Ma M, Xv Z, Chen X, Yang $X$ and Li P: TIM-3 promotes the metastasis of esophageal squamous cell carcinoma by targeting epithelial-mesenchymal transition via the Akt/GSK-3//Snail signaling pathway. Oncol Rep 36: 1551-1561, 2016.

30. Yi GZ, Liu YW, Xiang W, Wang H, Chen ZY, Xie SD and Qi ST3: Akt and $\beta$-catenin contribute to TMZ resistance and EMT of MGMT negative malignant glioma cell line. J Neurol Sci 367: 101-106, 2016. 